\title{
Drug Interactions Of Monoclonal Antibodies-Clinical Perspective
}

\author{
José M. Serra López-Matencio', Concepción Martínez Nieto', Alberto Morell Baladrón', Santos Castañeda* \\ 'Hospital Pharmacy Service, Hospital de la Princesa, IIS-Princesa, c / Diego de León 62; 28006-Madrid, Spain \\ ${ }^{2}$ Rheumatology Service, Hospital de la Princesa, IIS-Princesa, c / Diego de León 62; 28006-Madrid, Spain
}

\section{Article Info}

\section{Article Notes}

Received: February 09, 2018

Accepted: March 20, 2018

\section{${ }^{*}$ Correspondence:}

Dr. Santos Castañeda, Rheumatology Service, Hospital de la Princesa, IIS-Princesa, c / Diego de León 62; 28006-Madrid, Spain;

Fax: +34 91401 8752, E-mail: scastas@gmail.com; santos.castaneda@salud.madrid.org

(c) 2018 Castañeda S. This article is distributed under the terms of the Creative Commons Attribution 4.0 International License.

\section{Keywords:}

Biologics

Monoclonal antibodies

Chemical agents

Pharmacodynamics

Drug interactions

Pharmacologic interactions

\section{ABSTRACT}

Biological agents are used to treat a variety of diseases in many therapeutic areas, including oncology, hematology, rheumatology, gastroenterology, dermatology, neurology, respiratory diseases, hormone deficiency and infections. Since biologics constitute many of the recently approved new therapies, clinical research of drug-drug interactions with biologics has been discussed. Here, we present a personal view of drug-drug interactions with monoclonal antibodies, a predominant class of therapeutic biologics. In this line, we think that the interactions of biological agents with other chemical drugs represent an important issue, completely unknown and with potentially prominent clinical implications, that will have to be taken into account in coming years.

Health care practitioners around the world are facing an unprecedented challenge to improve care quality, prevent medical errors and maintain efficiency while simultaneously reducing costs of health systems. Identifying potentially harmful prescription drug interactions (before they have a chance to manifest themselves in the clinic) helps accomplish this goal in many ways. Unexpected clinical drug interactions can lead to a higher risk of adverse events and substantially reduce the probability of treatment success. Either of these outcomes can significantly increase resource utilization and unnecessarily prolong patient suffering and illness. It is because of that, a complete assessment of a new drug's potential for clinical drug interactions, including a thorough evaluation of any underlying pharmacokinetic or pharmacodynamic mechanisms, is always necessary ${ }^{1}$.

Nowadays, biological therapies play a crucial role in the pharmacotherapy of a variety of therapeutic disease areas, including those in hematology, oncology, rheumatology, gastroenterology, dermatology, neurology, hormone deficiency and infections ${ }^{2}$. Thus, clinicians are facing a new era of therapeutic treatment with profound differences in their chemical, pharmacokinetic and pharmacodynamic characteristics in comparison with the conventional therapies with small molecule drugs ${ }^{3}$. Chemically, therapeutic biologics are different than small chemical agents ${ }^{4}$ since they are mainly represented by (glyco) proteins composed of L-amino acids and various sugar molecules (Table 1).

There are several categories of therapeutic biologics, including monoclonal antibodies (mAbs), recombinant protein therapeutics 
Table 1. Main differences between chemical drugs and biological agents.

\begin{tabular}{|l|l|l|}
\hline \multicolumn{1}{|c|}{ Characteristics } & \multicolumn{1}{c|}{ Chemical } & \multicolumn{1}{c|}{ Biological } \\
\hline Type of synthesis & Produced by chemical synthesis & Produced from living cells \\
\hline Size & Small molecules & High molecular weight molecules \\
\hline Structure & Well defined & Complex and heterogeneous \\
\hline & Identical copies in each manufacturing process & Strongly dependent on the manufacturing process \\
\hline Manufacturing process relation & & \\
\hline & Well known every step of the process & Steps of the process owned by manufacturer \\
\hline Characterization & Completely characterized & Difficult to characterize \\
\hline Stability & Independent of external conditions & Sensitive to external conditions \\
\hline Immunogenicity & Mostly non immunogenic & Potentially immunogenic \\
\hline Modified from: Serra López-Matencio JM, et al. Reumatol Clin. 2016 (ref. 4). & \\
\hline
\end{tabular}

Table 2. Pharmacokinetic properties of chemical drugs and monoclonal antibodies.

\begin{tabular}{|l|l|l|}
\hline \multicolumn{1}{|c|}{ Parameter } & \multicolumn{1}{c|}{ Chemical } & \multicolumn{1}{c|}{ Monoclonal antibodies } \\
\hline Route of administration & Mainly oral & Parenteral \\
\hline Absorption & Reaches blood by capilars & $\begin{array}{l}\text { Reaches systemic levels mainly by lymphatic } \\
\text { system }\end{array}$ \\
\hline Distribution & Generally wide (reaches organs and tissues) & Small (difficult to reach organs and tissues) \\
\hline Metabolism & Metabolized by CYP450 and conjugation reactions & Catabolized to aminoacids \\
\hline Excretion & Mainly in liver and kidney & Reticuloendothelial system \\
\hline Clearance & Usually linear & Usually non linear \\
\hline Half life & Short (generally hours) & Long (weeks) \\
\hline Modified from: Serra López-Matencio JM, et al. Reumatol Clin 2016 (ref. 4). & \\
\hline
\end{tabular}

(e.g. therapeutic cytokines), hybrid and modified molecules (e.g. pegylated, glycoengineered, and fusion proteins), endogenous proteins/peptides (e.g. insulin) and others (e.g. gene transfer products and antisense oligonucleotides). Of all these categories, mAbs represent a predominant class of therapeutic protein biologics 5 . More than $150 \mathrm{mAbs}$ are in different stages of clinical development and, to date, 44 therapeutic mAbs have been approved for use by the FDA and EMA ${ }^{6}$. Although only a few formal drug-drug interaction studies have been performed with therapeutic mAbs, there are several recent studies giving excellent overviews of drug-drug interactions for therapeutic biologics including therapeutic $\mathrm{mAbs}^{7,8}$. In general, overall results of the literature review suggest that potential interaction effects between a therapeutic mAb and a coadministered small-molecule drug should be more thoroughly studied ${ }^{6}$.

A formal assessment of the drug-drug interaction potential of any investigational drug product requires multiple metabolic and pharmacokinetic evaluations. As for small-molecule drugs, investigating the drugdrug interaction potential is relatively easy. For mAbs, it is more complicated due to their complex nature. High molecular weight mAbs are often genetically engineered to demonstrate strong specificity for a particular human antigen target. Consequently, mAbs usually have few clinically relevant animal models (other than nonhuman primates) in which to conduct appropriate nonclinical studies. Similarly, clinical drug-drug interaction studies of mAbs with long elimination half-lives raise definite operational challenges since conventional crossover studies with adequate washout periods are difficult to conduct (Table 2). Added to all of this is the fact that the current regulatory guidance on the design and conduct of in vitro and in vivo drug-drug interaction studies applies more readily to small-molecule drugs ${ }^{9}$.

Very often, a drug-drug interaction results in a significant variation of the pharmacokinetic profiles caused by the common metabolic pathways involved in the disposition of the co-administered drugs. In particular, the inhibition or the induction of a particular isoform of cytochrome P450 (CYP450) or specific drug transporters by a single drug can dramatically affect the pharmacokinetics of a second drug when co-administered. Since the metabolism, distribution and elimination of mAbs are not mediated by CYP450 or drug transporters, mAbs are not expected to compete directly with chemically derived drugs. Thus, from a mechanistic perspective, the likelihood of direct drugmAbs interaction occurring during their co-administration is unlikely to be high ${ }^{10}$.

It has been suggested that some interactions between a mAb and a coadministered small-molecule drug could be attributed to a subsequent modulation of the activity between the $\mathrm{mAbs}$ and the $\mathrm{Fc} \gamma$ receptors on effector cells (i.e., lymphocytes, monocytes, macrophages and neutrophils) or to the concomitant effects of the small-molecule drug on the level of $\mathrm{Fc} \gamma$ receptor expression ${ }^{11}$. Other studies have asserted that certain mAbs may significantly affect 
the metabolism of a coadministered small-molecule drug through cytokine-induced inhibition of CYP3A $4^{12}$. Inflammation-evoked changes in drug transport proteins as a result of treatment with a mAb could also potentially affect the disposition of certain small-molecule drugs ${ }^{10}$.

Finally, in some cases where a concomitantly administered drug is processed by both CYP enzymes and transport proteins, the pharmacokinetic behavior may be greatly affected if immune-mediated changes related to $\mathrm{mAb}$ therapy were to occur simultaneously in the involved CYP enzymes and drug transport proteins. Recently, several combinations of mAbs have been studied in various stages of clinical development. A possible mechanism of interaction with these combinations would involve the FcRn-mediated recycling ${ }^{6}$. However, given the total amount of endogenous IgG of 50-100 g, the usual dose of most $\mathrm{mAbs}<10 \mathrm{mg} / \mathrm{kg}$ is not predicted to affect the total IgG plasma concentration ${ }^{13}$.

As mentioned above, the pharmacokinetics of mAbs is complex and differs from that of small molecule drugs. Their parenteral administration, the different pathways involved in their clearance and their long elimination halflife are the most pronounced pharmacokinetic distinctions (Table 2). The long half-life is partly the resultant of an important protective FcRn-based mechanism against degradation in the cell ${ }^{14}$. Monoclonal antibodies targeting a soluble antigen generally exhibit linear elimination whereas those targeting a membrane-bound antigen may exhibit non-linear elimination. The nonlinear clearance is due to the saturation of the target-mediated elimination of the mAbs. In addition, the immunogenicity of the mAbs is directly implicated in the clearance of mAbs, and their glycosylation pattern can influence this parameter ${ }^{15}$. Thus, and in contrast with the small molecule drugs, the mechanisms of pharmacokinetic interactions of mAbs are complex and currently are not well understood ${ }^{16}$.

Theoretically, because of their different disposal mechanism, the pharmacokinetic interaction between mAbs and small molecule drugs is expected to be minimal, as well as for the mAbs combination since their catabolic pathways are essentially not saturable. So far, the interactions of mAbs with each other and with small molecule drugs have not shown significant changes in systemic exposure ${ }^{17-20}$. Nevertheless, potentially clinically relevant interactions between mAbs and immunosuppressing drugs, such as methotrexate with infliximab, adalimumab and golimumab have already been documented ${ }^{21}$.

Small molecule drugs may affect the expression of the mAbs's target by altering their target-mediated clearance. Such case has been observed with statins and fibrates that induce the PCSK9 expression and thus the clearance of alirocumab and evolocumab, although this interaction is not considered clinically relevant ${ }^{22,23}$. Similarly, chemotherapeutic agents may reduce the tumor burden and hence the target-mediated clearance as discussed with trastuzumab, whose clearance increases with tumor size and therefore Her2 expression. In this light, the target-mediated clearance of $\mathrm{mAbs}$ seems to be the area that requires more investigation, and it is likely to have additional evidences in the near future.

In summary, the pharmacological interactions of biological agents are not well known. Because biological agents are not metabolized by cytochrome P450 enzymes and do not interact with cell membrane transporters, it is generally perceived that they are free from interactions with small molecule drugs. However, the clearance of biological agents varies depending on the modulation of the immune response or by either increasing or reducing the expression of target cells of the biological agents, which can occur by the action of multiple synthetic chemical agents. Furthermore, some biological agents may modify the metabolism of chemical drugs through their effects on the expression of P450 system enzymes.

In this clinical perspective, we provide an outline of the pharmacokinetics properties and pharmacologic interactions of biological drugs, focusing on mAbs, and how these can interact with chemical synthesis molecules. Thus, we believe knowledge of them is important for clinicians and affects multiple clinical specialties. However, the current information about relevant drug-mAbs interactions are not well described and more formal studies are needed in this field. Personally, we think that the interactions of biological agents with other chemical drugs represent an important issue, completely unknown and with potentially prominent clinical implications, that will have to be taken into account in the daily clinical management in coming years.

\section{Conflict of interests}

None declared.

\section{Funding}

None declared.

\section{Ackowledgements}

We thank Maria O'Driscoll for her technical support. Also, we want to express our gratitude to all the members of the Pharmacy and Rheumatology Divisions for their opinion and daily collaboration.

\section{References}

1. Zhou SF, Xue CC, Yu XQ, et al. Clinically important drug interactions potentially involving mechanism-based inhibition of cytochrome P450 3A4 and the role of therapeutic drug monitoring. Ther Drug Monit. 2007; 29(6): 687-710.

2. Ecker DM, Jones SD, Levine HL. The therapeutic monoclonal antibody market.Mabs.2015;7(1):9-14.DOI:10.4161/19420862.2015.989042. 
3. Langjahr P, Sotelo P. Present and future of therapeutic monoclonal antibodies. Mem Inst Investig Cienc Salud. 2016; 14(2): 110-121. DOI 10.18004/Mem.iics/1812-9528/2016.014(02)110-121.

4. Serra López-Matencio JM, Morell Baladrón A, Castañeda S. Biosimilars: A new scenario in biologic therapies. Reumatol Clin. 2016; 13(5): 287-293. DOI: 10.1016/j.reuma.2016.05.013.

5. Caorsi R, Federici S, Gattomo M. Biologic drugs in autoinflammatory syndromes. Autoimmun Rev. 2012; 12(1): 81-86. DOI: 10.1016/j. autrev.2012.07.027

6. Serra López-Matencio JM, Morell Baladrón A, Castañeda S Pharmacological interactions of therapeutic monoclonal antibodies. Med Clin (Barc). 2017 pii: S0025-7753(17)30845-X. DOI: 10.1016/j. medcli.2017.10.037

7. Mahmood I, Green MD. Drug interaction studies of therapeutic proteins or monoclonal antibodies. J Clin Pharmacol. 2007; 47(12): 1540-1554

8. Zhou H, Mascelli MA. Mechanisms of monoclonal antibody-drug interactions. Annu Rev Pharmacol Toxicol. 2011; 51: 359-372.

9. Zhang L, Zhang J, Strong $\mathrm{M}$, et al. A regulatory viewpoint on transporter-based drug interactions. Xenobiotica. 2008; 38(7-8): 709-724. DOI: 10.1080/00498250802017715.

10. Abdel-Razzak P, Loyer A, Fautrel JC, et al. Cytokines down-regulate expression of major cytochrome P-450 enzymes in adult human hepatocytes in primary culture. Mol Pharmacol. 1993; 44(4): 707-715.

11. Wang W, Wang EQ Balthasar JP. Monoclonal antibody pharmacokinetics and pharmacodynamics. Clin Pharmacol Ther 2008; 84(5): 548-558. DOI: 10.1038/clpt.2008.170.

12. Kim S, Ostor AJ, Nisar MK. Interleukin-6 and cytochrome-P450, reason for concern. Rheumatol Int. 2012; 32(9): 2601-2604. DOI: 10.1007/ s00296-012-2423-3

13. Blum PM, Phelps DL, Ank BJ, et al. Survival of oral human immune serum globulin in the gastrointestinal tract of low birth weight infants. Pediatr Res. 1981; 15(9): 1256-1260.
14. Roopenian DC, Akilesh S. FcRn: the neonatal Fc receptor comes of age. Nat Rev Immunol. 2007; 7(9): 715-725. DOI: 10.1038/nri2155.

15. Waldmann TA, Strober W. Metabolism of immunoglobulins. Prog Allergy. 1969; 13: 1-110.

16. Keizer RJ, Huitema AD, Schellens JH, et al. Clinical pharmacokinetics of therapeutic monoclonal antibodies. Clin Pharmacokinet. 2010; 49(8): 493-507. DOI: 10.2165/11531280-000000000-00000.

17. Baert F, Noman M, Vermeire S, et al. Influence of immunogenicity on the long-term efficacy of infliximab in Crohn's disease. N Engl J Med. 2003; 348(7): 601-608.

18. Seitzand K, Zhou H. Pharmacokinetic drug-drug interaction potentials for therapeutic monoclonal antibodies: reality check. J Clin Pharmacol. 2007; 47(9): 1104-1118.

19. Weisman MH, Moreland LW, Furst DE, et al. Efficacy, pharmacokinetic, and safety assessment of adalimumab, a fully human anti-tumor necrosis factor-alpha monoclonal antibody, in adults with rheumatoid arthritis receiving concomitant methotrexate: a pilot study. Clin Ther. 2003; 25(6): 1700-1721.

20. Seitz K, Zhou H. Pharmacokinetic drug-drug interaction potentials for therapeutic monoclonal antibodies: reality check. J Clin Pharmacol. 2007; 47(9): 1104-18.

21. Zhou H, Jang H, Fleischmann RM, et al. Pharmacokinetics and safety of golimumab, a fully human anti-TNF-alpha monoclonal antibody, in subjects with rheumatoid arthritis. J Clin Pharmacol. 2007; 47(3): 383-396. DOI: $10.1177 / 0091270006298188$

22. EMA, Evolocumab, Summary of product characteristics. http:// wwwemaeuropaeu/docs/en GB/document library/EPAR ProductInformation/human/003766/WC500191398pdf (accessed April 2016).

23. EMA, Alirocumab, Summary of product characteristics. http:// wwwemaeuropaeu/docs/en GB/document library/EPAR - Product Information/human/003882/WC500194521pdf (accessed April 2016). 